\title{
A RE-EVALUATION OF MOBILE COMMUNICATION TECHNOLOGY: A THEORETICAL APPROACH FOR TECHNOLOGY EVALUATION IN CONTEMPORARY DIGITAL LEARNING
}

\author{
OnUr YUMURTACI \\ Faculty of Communication Sciences \\ Anadolu University \\ Eskisehir, Turkey
}

\section{ABSTRACT}

We live in an age of continual technological development. Rapidly developing technologies have found use in nearly all aspects of life. As such, it is understandable that technology has also infiltrated the field of education. Information and Communication Technology (ICT) has provided us with the technical underpinnings for distance and lifelong learning. Our understanding of learning has shifted in accordance with the capabilities of technology in such a way that we have had to re-think our approach to learning as a whole. Connectivism is one such approach which aims to re-consider learning within the scope of our relatively new, networked social structure. The Theory of Connectivism relies heavily on what we are technically capable of, and therefore it is also important that we re-evaluate our approach to the technology we use in learning. Due to these aforementioned shifts in our approach towards learning, this study aims to provide a theoretical framework for the re-evaluation of the technology we utilize in connectivist learning; more specifically, how to evaluate our perception of mobile communication technology. A combination of the Technology Acceptance Model and the Media Naturalness Theory is proposed for the evaluation of user perception of mobile communication technology, and the implications of possible outcomes of this reevaluation are discussed with regards to connectivist learning and education as a whole.

Keywords: Connectivism, communication technology, media naturalness theory, technology acceptance model.

\section{INTRODUCTION}

The $21^{\text {st }}$ century has brought many innovations to various fields, many of which have been initiated by technological developments. Even before the advent of technologies such as the Internet and voice over IP, it was speculated that communication technologies would geographically reduce the world to a "global village" by eliminating the constraints of time and space (McLuhan, 2003). It is therefore understandable that the rapid developments in communication technologies have had a drastic influence in our social structures. Considering the changes and possibilities enabled through ICTs, Castells (2004) proposed that our social structure has moved away from hierarchies and towards that of a "network society". This change in social structure is caused by the fact that contrary to the developments of the industrial age, communication technology has made information a greatly sought commodity and has inadvertently caused a shift towards a "techno-economy" paradigm in which knowledge carries the greatest value. Castells (2004) states that within this techno-economy, the only way for societies, establishments and individuals to flourish is through an educational approach in which individuals learn to rapidly acquire and develop new skills that allow them to stay current throughout their lives. 
The field of education has also been influenced by the development of ICTs and the affordances they allow. The basic concept of distance education relies on the fact that geographical distance is no longer a limiting factor. This being the case, theories developed towards traditional modes of education may be insufficient in their explanatory power. Siemens (2005) proposes the Theory of Connectivism as a new approach to learning in a digital age. This approach takes into account the technological basis of how we interact as a society and will be discussed further in following sections. The main focus of this study, however, is the issue of user perceptions regarding mobile communication technologies. These technologies were selected for this study due to their relevance regarding the "anywhere, anytime" approach to connectivist learning. Following a short description of Connectivism and connectivist learning, the Technology Acceptance Model and Media Naturalness Theory are discussed as viable approaches to the evaluation of user perceptions of mobile communication technologies for use in connectivist learning.

\section{CHANGES IN KNOWLEDGE AND LEARNING: CONNECTIVISM}

With the advent of technologies that significantly manipulate the educational process, the explanatory power of behaviorism, cognitivism, and constructivism may not be sufficient to analyze the learning that takes place today. This is the basic argument behind proponents of Connectivism, which aims to provide a learning theory that incorporates the networked nature of today's society along with the technological developments that allow us to maintain this networked structure (Siemens, 2006).

Connectivism is based on the concept that along with the changes that took place in society towards a networked arrangement, learning itself has transformed in that access to information is of paramount importance. Siemens (2006) argues that the sheer amount of information is too much for any one person to handle and therefore it is of even greater importance that we know how and where to access information. Following this logic, connectivist learning implies that information rests in nodes of networks (where nodes are sources of specialized information, human or otherwise) and knowledge itself has adapted to these circumstances as it resides in the network itself, thereby enabling continuous learning in formal and informal settings (Siemens, 2006). Siemens (2005) states that traditional approaches to learning treat knowledge as an internal or internalizable object, and thus these approaches fail at analyzing the rapidly changing concept, content and landscape of education and learning regarding knowledge. Considering the constantly shifting nature of society, then, Siemens (2006) argues that arriving at any constant definition of knowledge as a concept renders it useless for diverse implementation, thereby indicating that traditional approaches to learning also fail to account for the diverse methods in which we learn. In this regard, Siemens (2005) draws attention to some significant trends in learning:

$>$ Many learners will move into a variety of different, possibly unrelated fields over the course of their lifetime.

$>$ Informal learning is a significant aspect of our learning experience. Formal education no longer comprises the majority of our learning. Learning now occurs in a variety of ways - through communities of practice, personal networks, and through completion of work-related tasks.

$>$ Learning is a continual process, lasting for a lifetime. Learning and work related activities are no longer separate. In many situations, they are the same.

$>$ Technology is altering (rewiring) our brains. The tools we use define and shape our thinking.

$>$ The organization and the individual are both learning organisms. Increased attention to knowledge management highlights the need for a theory that attempts to explain the link between individual and organizational learning. 
$>$ Many of the processes previously handled by learning theories (especially in cognitive information processing) can now be off-loaded to, or supported by, technology.

$>$ Know-how and know-what is being supplemented with know-where (the understanding of where to find knowledge needed).

Based on these trends in learning and drawing from the aforementioned changes in the landscape of knowledge, Siemens (2005) states that connective learning addresses these issues by proposing a learning approach which allows for constant adaptation through always current information accessible through connections. The principles of Connectivism are stated as follows (Siemens, 2005):

$>\quad$ Learning and knowledge rests in diversity of opinions.

$>\quad$ Learning is a process of connecting specialized nodes or information sources.

$>$ Learning may reside in non-human appliances.

> Capacity to know is more critical than what is currently known.

$>$ Nurturing and maintaining connections is needed to facilitate continual learning.

$>$ Ability to see connections between fields, ideas, and concepts is a core skill.

$>$ Currency (accurate, up-do-date knowledge) is the intent of all connectivist learning activities.

$>$ Decision-making is itself a learning process. Choosing what to learn and the meaning of incoming information is seen through the lens of a shifting reality. While there is a right answer now, it may be wrong tomorrow due to alterations in the information climate affecting the decision.

In accordance with these principles, it can be argued that technological developments in the field of communication are an important aspect of the fulfillment of connectivist learning.

\section{CHANGES IN SOCIETY AND TECHNOLOGY}

In the face of changing circumstances surrounding our understanding of knowledge and learning, it is paramount that we also understand the technological innovations that not only permit the aforementioned developments in learning, but also how they have interacted with society thereby transforming our personal connections with technology. The rapid development in digital technology has even birthed a new generation whom grew up immersed in technology: digital natives (Prensky, 2001). As opposed to digital immigrants, digital natives have developed their awareness regarding technology on a much more personal level, thereby reaching a level of digital literacy that is arguably difficult for digital immigrants to achieve (Prensky, 2001; Irving \& English, 2011). This has also impacted the personal nature of technology, as in a knowledge-economy based society, constant access is a key aspect of the modern networked individual.

One of the most significant developments in digital communication technology has been the addition of mobility to the hardware utilized for communication. This additional aspect of technology has spawned "portable" technology, or the concept of a person carrying technology on their person. While the terminology utilized to denote this concept has evolved into the utilization of the term "mobile", the basic ability to communicate anywhere via devices we can carry on our persons without major hindrance to our everyday lives has massively transformed our approach to time and space. Mobility has been a second stage in the communication revolution that spawned the network society, by increasing the level of disconnection between ourselves and space/time (Kakihara \& Sorensen, 2001). Our immersion with technology has reached such heights that through mobile communication technology, a class of people who utilize these technologies to stay constantly connected regardless of geography or medium has risen: digital nomads (Kakihara \& Sorensen, 2001). Digital nomads who take advantage of their network connectedness gain personal mobility yet carry their interactive lifestyles on their persons 
through the utilization of wireless, mobile communication hardware and network services and software (Sorensen, 2002). As society reaches a previously unforeseen level of connectedness and flexibility, the deeply personal aspect of mobile technology begins to surface.

To better understand the personal aspect of mobile technology, along with how deeply integrated it may become in connectivist mobile learning environments, we must consider the definitive difference between that which is "mobile", and that which is "portable". In this regard, Jon Agar (2013) draws attention to the innate and personable importance of each object we choose to carry upon our persons. Just as one would carry a comb if they place importance on their personal appearance, the notion of the ability to communicate beyond the constraints of time and space - while in motion - is most likely the appeal that has led users to carry first or second generation mobile phones. The distinction of being able to communicate "while in motion" is most likely the driving cause of the proliferation of mobile communication technologies in our daily lives, ensuring a constant and permanent connection to individuals, information, and society. Agar also notes that mobile phones have drawn attention as radical new personal devices in that they replace single-function items, combining their utility into a single device, exemplifying this as follows: while a lawnmower is arguably a single-function device, it may also be used to prop doors open. In the case of the mobile phone, smart phones are basically fully functioning computers, thereby being capable of all the functions that computers are capable of. As a result of this flexibility, Agar argues that one would therefore want to be able to carry these capabilities with oneself. Based on this approach, Agar provides three distinct definitions regarding the "three concentric rings of personal technologies" (2013):

$>$ The outer ring: Items "owned" by an individual that are for the most part immobile and do not move with them, such as desktop computers or refrigerators.

> The middle ring: Technologies which are "portable" in that they may be carried by an individual when necessary, but their mobility requires effort or exertion that may be an inconvenience, such as laptop computers.

$>$ The inner ring: Items carried without effort and on one's person such as smartphones, the weight of which may be disregarded due to their utility, making them "intimate" technologies.

These "rings" provide a method of defining mobile technologies based on a constant: the individual. As such, these definitions will most likely also remain true throughout various iterations and developments of technologies, no matter how drastic the change and transformation. In reference to the use of mobile technology for connectivist learning, however, it may be argued that the defining characteristic of mobile technologies and especially smart phones is that they have expanded beyond their intended role of mobile communication, and through the convergence of various multimedia features, "is no longer simply a phone" (Westlund, 2008).

Demographic studies conducted on mobile technology use indicate that while there is an apparent majority in mobile technology use in relatively younger generations, technology adoption is not limited to digital natives (Zickuhr, 2011). Horrigan (2007) studied the qualities of various user groups regarding ICT use in society, and repeated his study with emphasis on mobile and stationary technology, revealing that while youths primarily utilized ICTs to their fullest potential, an important portion of active users are somehow related with higher education (in the form of active studentship, or as graduates) (Horrigan, 2009). Network (or with respect to Horrigan's study, Internet) access was determined to be a determining factor in mobile technology preference and considering the data regarding users who utilize mobile technology to its fullest (referred to as Elite Users) overlaps with previous data indicating high technology utilization among students, 
a better understanding of the perception of mobile technology could provide useful for a better understanding of its current and potential uses. Mobile technology tends to be very "personal" in nature, and it can be argued that studies conducted on the relationship between technology and society on an organizational or institutional level fail to grasp the personal experience and distinct, personalized needs utilization of technology (Wiredu, 2007). To address this issue, and to better understand the relationship between mobile technology and its users this study proposes a combination of approaches to technology use: a combination of the Technology Acceptance Model (Davis, 1989) and the Media Naturalness Theory (Kock, 2005).

\section{A NEW APPROACH TO EVALUATION}

The concept of evaluation covers a broad context in the realm of learning and education. Studies of evaluation cover issues from policy changes to specialized course content, teaching and learning strategies to the implementation of learning tools. As a field developing in collaboration with ICTs, distance education in particular draws benefits from the evaluation of the ICTs currently and prospectively implemented in learning. The concept of evaluation itself, however, has been limited in that as far as the evaluation of educational and learning technologies are concerned, emphasis is placed (arguably rightfully) on the learning outcomes associated with the use of these technologies, along with other key considerations for the evaluation of learning technology as per indicated by Oliver (2000). This approach, however, is arguably less applicable in the case of connectivist learning, as the act of learning itself relies heavily on the capability of the learner to create and maintain new connections through which they can access and create knowledge. Connectivist learning relies on the accessibility of information, and the ubiquity of the tools to gain and maintain that access. The act of learning itself is therefore heavily dependent on the use of technology by the learner, along with the level of communication (and therefore access) provided to the learner by this technology. It is due to this distinction that this study draws attention to the evaluation of mobile communication technologies as primarily tools of communication, as such an evaluation would provide insight into the possible strengths and weaknesses of these tools in connectivist learning environments. Similarly, Motiwalla (2007) proposes a framework and evaluation for mobile learning, indicating that the intended use for such a framework or method of evaluation is to "provide the requirements to develop $\mathbf{m}$-learning applications that can be used to complement classroom or distance learning"; yet continues to state that " Learning on wireless/handheld devices will never replace classroom or other electronic learning approaches.", thereby somewhat negating the underlying potential of communication technologies in learning. The proposed framework focuses distinctly on mobile communication technologies as handheld apparatus utilized to access supplementary learning materials outside of the classroom environment, disregarding the possibility that learning may take place anywhere, any time. Such limitations in the understanding of the technologies utilized in learning lie at the core of a lack of theoretical models on which assessment and evaluation of these technologies may depend. Either by focusing on the learning process and disregarding the tools utilized, or by focusing only on the possible learning scenarios afforded by these tools, currently established frameworks and models overlook the potential for utilizing technologies in previously unforeseen ways. This is the inherent reason that this study emphasizes the evaluation of mobile technologies primarily as tools of communication. If the Theory of Connectivism is to be adhered to, knowledge may lie in both the tools and in the users, and access to these resources lie in learners' ability to create and maintain connections to 
these nodes. Taking into account the "mobile" aspect of learners, evaluating mobile communication technologies that may be used for learning purposes must start by evaluating their prospective uses - how users themselves perceive these technologies along with their ability to successfully achieve their intended purpose - as mediums of communication.

Within the context of this study, the proposed two-pronged approach to the evaluation of mobile communication technology incorporates the Technology Acceptance Model, and the Media Naturalness Theory, both of which are further discussed below. The Technology Acceptance Model has been previously utilized individually in evaluative capacities (Lee et al, 2003) whereas the Media Naturalness Theory is a proposed extension of the Media Richness Theory, which has prominently been used in previous research in communications - though with distinct shortcomings related to it's capacity regarding new communication technologies (El-Shinawy \& Markus, 1997). As of the writing of this article, no previous research has been encountered in which both approaches are combined for the distinct purpose of evaluating mobile communication technologies. It is believed that the explanatory power of the combination of these two approaches would provide a more substantial and dependable framework for such an evaluation.

\section{TECHNOLOGY ACCEPTANCE MODEL}

One of the most widely utilized explanatory models regarding the relationship between society and technology is the Technology Acceptance Model (TAM). Davis (1989), recognizing the issues regarding user acceptance in technology and the lack of high quality measures for user acceptance, developed TAM based on two main constructs:

$>$ Perceived Usefulness: The degree to which a person believes that using a particular system would enhance his or her job performance.

$>$ Perceived Ease of Use: The degree to which a person believes that using a particular system would be free of effort.

Davis expands on perceived usefulness, stating that individuals are more likely to use or not use a technology based on their belief of whether or not it will do a job better. Regarding perceived ease of use, Davis indicates that even if potential users believe in the usefulness of a technology, they may also believe that the effort and exertion to use that technology may surpass the performance gains to be obtained through use, thereby believing the system to be too difficult to use.

TAM has been criticized for reducing the complex mechanism of technology acceptance to two seemingly vague measures. The prominence of TAM in studies, however, could be considered an indication of its versatility in the matter, and research has shown that TAM's measures retain their efficacy under a variety of circumstances (Lee et al, 2003). In its eventuality, TAM has evolved into the Unified Theory of Acceptance and Use of Technology, yet studies indicate that the involvement of further measures upon the two bases of TAM introduces unnecessary complexity relating to the additional measures (Bagozzi, 2007). An intermediary iteration of TAM, called TAM2, which incorporates social factors and cognitive tools in addition to the two basic measures of TAM could be considered as a viable approach to technology acceptance and use. Studies, however, have indicated that the incorporation of factors such as Usage Attitude did not have a determining effect on intended or actual use (Wu \& Wang, 2005). Within the scope of this 
study, it has been decided that educational theory takes precedence regarding the social aspects of technology use for learning and is the defining reasoning behind the selection of TAM as the first part of the proposed two-pronged approach to technology use.

\section{MEDIA NATURALNESS THEORY}

Media Naturalness Theory (MNT) is based on the evolutionary theory of Charles Darwin in that it attempts to explain our communication preferences based on are existing communicative apparatus: our senses. While developing MNT, Ned Kock took into consideration the shortcomings and limitations of the Media Richness Theory (MRT) (Kock, 2005). The fundamental flaw that Kock presents regarding MRT is that there are many studies supporting the media richness hypothesis that fail to explain peoples' preference towards face-to-face interaction, stemming from the fact that MRT fails to explain our lenience towards "rich" media (Kinney \& Dennis, 1994; Kock, 2005; Kock 2001). Studies have also indicated that MRT fails to retain its validity when studying new communication technologies (El-Shinawy \& Markus, 1997). Kock provides a theoretical basis for his argument for media "naturalness" as a primary precedent for our selection of communication media through our natural tendency towards face-to-face communication through the evolution of our biological apparatus. From this basis, MNT has five fundamental elements in defining a communication medium relative to face-to-face communication (Kock, 2005):

$>$ A high degree of colocation, which would allow the individuals engaged in a communication interaction to see and hear each other.

$>\quad A$ high degree of synchronicity, which would allow the individuals engaged in a communication interaction to quickly exchange communicative stimuli.

$>$ The ability to convey and observe facial expressions.

$>\quad$ The ability to convey and observe body language.

$>\quad$ The ability to convey and listen to speech.

Kock (2005) states that communication that takes place based on these factors may accommodate the elements of face-to-face communication, and adapting these elements to electronic communication environments can provide an experience closest to face-toface communication. MNT provides a method for evaluating communication preferences through psychological and physiological dimensions, and its explanatory power is based on our evolutionary tendency towards face-to-face communication. As such, MNT combined with TAM from the previous section provides us with a flexible yet decisive theoretical method for evaluating mobile communication technology.

\section{THEORETICAL MATRIX OF MOBILE COMMUNICATION TECHNOLOGY EVALUATION}

In accordance with the measures of both TAM and MNT portrayed above, these two approaches may be combined to provide a comprehensive strategy for the evaluation of communication technologies. The combination of these measures are portrayed in Table 1 below: 
Table 1. Theoretical Matrix.

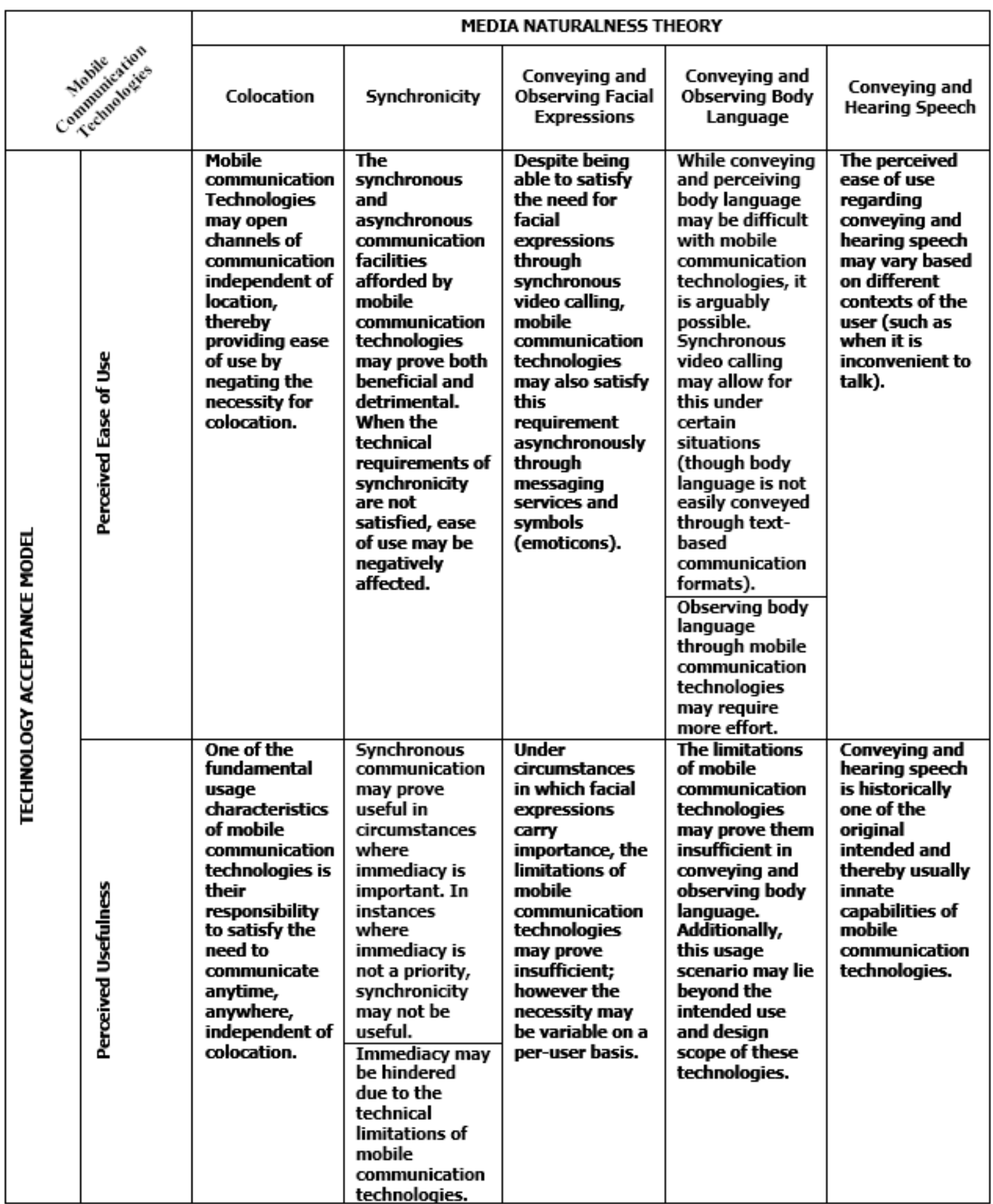


As can be observed in Table 1, the combination of the key elements of both TAM and MNT reveal certain emerging themes from which an evaluation of mobile communication technologies may be conducted. Through this matrix, the intersection of each of the proposed measures of each theory provide a guide in which tangential correlations are formed. This, in turn, combines the explanatory power of both theories into a flexible frame of reference from which both qualitative and quantitative methods of measurement may be derived. Additionally, this frame of reference may also be used to develop a checklist with regard to the design and implementation of mobile communication technologies in learning environments, both as an a priori element to establish the requirements expected of mobile communication technologies in learning environments, and as a posteriori reference to assess the achievement of predetermined goals through mobile communication technology.

\section{CONCLUSION}

At this point, the matters discussed above converge regarding issues being faced by the field of education. Learning has undeniably been influenced by developments in technology, as those developments have also manipulated society as a whole, and individuals on a personal basis. Learning has transcended into a realm combining formal and informal learning, in which knowledge and information have become matters of accessibility. The Theory of Connectivism provides some perspective on the matter in that it takes into account the shifting nature of learning and knowledge in accordance with contemporary modes of learning and information access. To accommodate this approach, we are also in need of a method to evaluate the technologies involved in the process. The departure from traditional learning forms brings with it a departure from traditional learning mediums and incorporates a plethora of technologies capable of serving various needs for today's learner.

Emerging technologies have proven exemplary in the opportunities they provide. Constant network access accompanied by Wikis and social media has brought information accessibility to a whole new level, drastically changing the mediation of information and our consumption of data and information in daily life. Connectivist learning dictates that the skills needed to efficiently utilize the affordances these new technologies allow us are rapid adaptation and connection-forming. While digital natives and digital nomads may not face that many issues regarding technology utilization, digital immigrants are confronted with a level of information throughput they may have never faced before. On a fundamental level, we must be capable of understanding the process of media selection and technology adoption before we can effectively utilize emerging technologies in learning spaces. The two-pronged approach of TAM and MNT proposed in this study introduces a method for evaluating technology in a way that will help us understand the opportunities, benefits, and shortcomings of the technology we seek to grasp and utilize in learning. This proposed method of evaluating mobile communication technologies allows us to gather essential information regarding how much use these technologies are likely to see in a learning context along with how effective they will be at conveying the information necessary for learning to take place. This understanding, in turn, could allow us to adapt the conception, design, implementation, and evaluation of technology in learning for beneficial and cost-effective results, allowing us to make these evaluations not as a learning outcome after everything is said and done, but before implementation as a precursor to learning taking place.

In the battle to better understand and incorporate learning and life-long education, understanding the tools we use to learn - consciously or otherwise - is an elemental step in the constant development process of tailoring learning to society. As such, while this study focuses mainly on the application of the proposed two-prong approach to technologies that are intended for utilization in connectivist learning environments, it is also plausible and in fact suggested that this approach may prove beneficial in assessing the technological infrastructure of all forms of learning in which mobile technologies are to 
be utilized. This includes but is not limited to mobile learning, e-learning, u-learning, and distance and open education, and is especially true considered the current ubiquity of mobile communication technologies in society.

The opportunities afforded by the development of mobile technologies are truly astounding, and these technologies find application in a plethora of educational situations ranging from formal to informal, structured to unstructured. They allow for the presentation of learning content and application of standardized testing beyond the constraints of time and space, yet also allow the opportunity for learners to manage their own learning space and pace. It is precisely due to these opportunities afforded by mobile technology that the educational community as a whole develops a better understanding of the underlying technology and how it is perceived and utilized by the users and learners.

Author's Note: Article derived from theoretical framework of Ph.D. thesis.

\section{BIODATA and CONTACT ADDRESSES of AUTHOR}

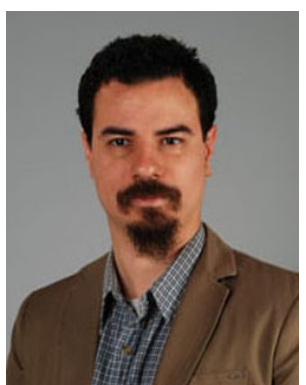

Dr. Onur YUMURTACI is a research assistant at the Faculty of Communication Sciences of Anadolu University in Eskisehir, Turkey. He completed his Ph.D. in $\mathbf{2 0 1 5}$ on attitudes towards mobile communication technologies, and has conducted, published, and presented research in the field of communications. His research interests mainly focus on the social aspects of mobile technology. As such, his current research endeavors revolve around $m$-Learning, u-Learning, and the audiovisual aspects of mobile communication technologies.

\section{Dr. Onur YUMURTACI}

Anadolu University Faculty of Communication Sciences

Department of Film and Television

Anadolu University, 26470 Tepebasi, Eskisehir, Turkey

Phone: +90 (222) 3350580 ext: 5280

E-mail: oyumurtaci@anadolu.edu.tr

\section{REFERENCES}

Agar, J. (2013). Constant Touch: A Global History of the Mobile Phone. London: Icon Books.

Castells, M. (2004). The Information Age: Economy, Society \& Culture. Oxford: Blackwell Publishers.

Bagozzi, R. (2007). The Legacy of the Technology Acceptance Model and a Proposal for a Paradigm Shift. Journal of the Association for Information Systems, 8 (4), 244254.

Davis, F. (1989). Perceived Usefulness, Perceived Ease of Use, and User Acceptance of Information Technology. MIS Quarterly, 13 (3), 319-140.

El-Shinnawy, M., Markus, L. (1997). The poverty of media richness theory: explaining people's choice of electronic mail vs. voice mail. International Journal of HumanComputer Studies, 45, 443-467.

Horrigan, J. (2007). A Typology of Information and Communication Technology Users. American Life Project. Retrieved on December 16, 2011 from www.pewresearch.org 
Horrigan, J. (2009). The Mobile Difference. Retrieved on December 16, 2011 from www.pewresearch.org

Irving, J., English, M. (2011). Community in Cyberspace: Gender, Social Movement Learning, and the Internet. Adult Education Quarterly, 61 (3), 262-278.

Kakihara, M., Sorensen, C. (2001). Expanding the 'Mobility' Concept. SIGGROUP BULLETIN, $22(3), 33-37$.

Kinney, S., Dennis, A. (1994). Re-evaluation media richness: Cues, feedback, and task. Hawaii International Conference on Systems Sciences, 21-30.

Kock, N. (2001). The Ape That Used Email: Understanding E-Communication Behavior Through Evolution Theory. Communications of the Association for Information Systems, 5(3), 1-29.

Kock, N. (2005). Media Richness or Media Naturalness? The Evolution of Our Biological Communication Apparatus and Its Influence on Our Behavior Toward ECommunication Tools. IEEE Transactions on Professional Communication, 48 (2), 117-132.

Lee, Y., Kozar, K., Larsen, K. (2003). The Technology Acceptance Model: Past, Present, and Future. Communications of the Association for Information Systems, 12, 752-780.

Mcluhan, M. (2003). Understanding Media. California: Gingko Press.

Motiwalla, L. F. (2007). Mobile learning: A framework and evaluation. Computers \& Education, 49, 581-596.

Oliver, M. (2000). An Introduction to the Evaluation of Learning Technology. Journal of Educational Technology \& Society, 3 (4), 20-30.

Prensky, M. (2001). Digital Natives, Digital Immigrants. On The Horizon, 9 (5), 1-6.

Siemens, G. (2005). Connectivism: A Learning Theory for the Digital Age. The International Journal of Instructional Technology \& Distance Learning. Retrieved on January 16, 2012 from http://www.itdl.org/Journal/Jan_05/article01.htm

Siemens, G. (2006). Knowing Knowledge. Retrieved on January 12, 2012 from http://www.elearnspace.org/KnowingKnowledge_LowRes.pdf

Sorensen, C. (2002). Digital nomads and mobile services. Receiver, 6, 1-6.

Westlund, O. (2008). From Mobile Phone to Mobile Device: News Consumption on the Go. Canadian Journal of Communication, 33 (3), 443-464.

Wiredu, G. (2007). User appropriation of mobile technologies: Motives, conditions and design properties. Information and Organization, $17(2), 110-129$.

Wu, J., Wang, S. (2005). What drives mobile commerce? An empirical evaluation of the revised technology acceptance model. Information \& Management 42, 719-729.

Zickuhr, K. (2011). Generations and their gadgets. Retreived on January 16, 2012 from www.pewresearch.org 